ISSN: 0210-1696

DOI: http://dx.doi.org/10.14201/scero20154623155

\title{
LA DETECCIÓN E INTERVENCIÓN TEMPRANAS EN MENORES CON TRASTORNO DEL ESPECTRO AUTISTA
}

\section{Early Detection and Intervention for Autism Spectrum Disorders in Toodlers}

Patricia García Primo

Instituto de Investigación de Enfermedades Raras. Instituto de Salud Carlos III. Madrid. Monforte de Lemos, 5. 28029 Madrid

pgarciaprimo@isciii.es

Manuel Posada DE LA PAZ

Instituto de Investigación de Enfermedades Raras. Instituto de Salud Carlos III. Madrid

M. ${ }^{a}$ Victoria Martín Cilleros

Instituto Universitario de Integración en la Comunidad (INICO). Facultad de Educación. Universidad de Salamanca

José Santos Borbujo

Departamento de Obstetricia, Ginecología y Pediatría. Facultad de Medicina. Universidad de Salamanca

Gloria Bueno CARrera

Departamento de Psiquiatría, Psicología, Medicina Legal e Historia de la Ciencia. Facultad de Medicina. Universidad de Salamanca

Ricardo CANAL BEDiA

Instituto Universitario de Integración en la Comunidad (INICO). Facultad de Educación. Universidad de Salamanca

Recepción: 31 de julio de 2014

Fecha de aceptación definitiva: 25 de marzo de 2015

Biblid. [0210-1696 (2015) vol. 46 (2), n. ${ }^{\circ} 254$, abril-junio; 31-55]

RESUMEN: La importancia de la detección y de la intervención precoz en los trastornos del espectro autista (TEA) ha adquirido gran interés tanto para las sociedades profesionales como por la administración sanitaria. Con el propósito de facilitar la puesta en marcha de dispositivos de detección y de atención e intervención a edades tempranas de estos niños, es necesaria una información actualizada y sintetizada sobre los procedimientos de cribado y las técnicas de atención temprana para niños con TEA, reflejando 
los aspectos más relevantes que se deduzcan de las experiencias y estudios llevados a cabo hasta el momento. Tras la revisión realizada en nuestro estudio sobre las experiencias de detección precoz de autismo, se obtiene un grupo de consideraciones importantes, para realizar análisis críticos de programas de cribado en marcha así como recomendaciones para experiencias futuras. Así mismo, la revisión de los estudios sobre la eficacia de los programas de atención temprana nos proporciona una visión esperanzadora, indicando que éstos son cada vez más frecuentes, sistemáticos y metodológicamente más apropiados. Llevamos mucho camino recorrido, pero aún queda mucho por hacer.

Palabras Clave: autismo; detección; intervención temprana; tratamiento.

AвSTRACT: The importance of screening and early intervention in autism spectrum disorders (ASD) has gained great interest not only for the scientific societies but also for the health and welfare systems. In order to facilitate the selection and implementation of the best screening procedures and intervention programs for children at early ages and their families, it is necessary an updated and synthesized review of the experiences and studies carried out so far that would show the most relevant aspects of these methods. After carrying out this revision, several important considerations were extracted aiming for critical analysis of screening programs in place, as well as recommendations for future experiences. In addition, the review of studies on the effectiveness of early intervention programs provides an encouraging scenery, indicating that these are becoming more frequent, systematic and methodologically appropriate. We have walked a long journey, but much work remains to be done.

KEY WORDS: autism; screening; early intervention; treatment.

\section{Introducción}

OS TRASTORNOS DEL ESPECTRO AUTISTA (TEA) se caracterizan por la presencia de déficits persistentes en comunicación e interacción social junto con patrones repetitivos y restringidos de conductas, actividades e intereses, que se manifiestan durante el periodo de desarrollo temprano, causando dificultades clínicamente significativas en la vida social y ocupacional. El funcionamiento de las personas con autismo y los resultados que el tratamiento logre en cada caso se ven afectados en gran medida no solo por la gravedad de los síntomas básicos que presente el individuo, sino que con frecuencia también otros aspectos, como el funcionamiento intelectual, la presencia/ausencia de lenguaje, la irritabilidad, anormalidades sensoriales o hiperactividad, influyen en el desarrollo posterior de cada persona afectada.

Hoy en día se considera que los TEA son un importante reto para la salud pública y para la sociedad en general, ya que la mayoría de los estudios sobre prevalencia proporcionan estimaciones que están en torno a 6 casos por cada 1.000 (Elsabbagh et al., 2012; Kogan et al., 2009) aunque estudios más recientes sugieren una prevalencia aún mayor, con aproximadamente 11 casos por cada 1.000 niños entre las edades de 3 y 17 años (Kogan et al., 2009). En España estaríamos hablando de algo más de 70.000

(C) Ediciones Universidad de Salamanca

Siglo Cero, vol. 46 (2), n. ${ }^{\circ}$ 254, 2015, abril-junio, pp. 31-55 
niños y adolescentes afectados por TEA. Tomando en consideración estas cifras la necesidad de la detección y de tratamientos eficaces para los TEA nunca ha sido tan evidente y es comprensible que tanto la administración sanitaria como las sociedades profesionales y las organizaciones de apoyo a las personas con TEA recomienden y reclamen la detección precoz y la atención temprana.

Esta revisión tiene el propósito de proporcionar información actualizada sobre los procedimientos de detección precoz y sobre las técnicas de atención temprana para niños con TEA para ayudar a que los profesionales puedan llevar a cabo una detección y diagnóstico más tempranos y eficientes y para que puedan hacer recomendaciones apropiadas a las familias cuando identifican a niños pequeños con TEA en su práctica clínica.

\section{Sistemas de cribado para la detección precoz de los TEA}

Desde principios de los años 90 del pasado siglo hasta la actualidad se ha desarrollado una gran variedad de estudios de detección precoz del autismo. La variabilidad de este tipo de programas nos obliga a revisar las experiencias llevadas a cabo que hayan sido publicadas desde los inicios de este siglo de modo que se pueda llegar a conclusiones sobre los aspectos importantes a considerar en este campo de la detección precoz. De la revisión de experiencias publicadas se obtiene el siguiente grupo de consideraciones importantes que pasamos a describir a continuación.

\section{Enfoque cualitativo de los resultados con la berramienta}

La mayoría de los estudios sobre instrumentos de cribado de TEA refieren los mismos valores estadísticos para medir su validez y fiabilidad. La sensibilidad (proporción de casos con el trastorno que son identificados como de riesgo) y la especificidad (proporción de niños sin el trastorno que son identificados como de no riesgo) son consideradas, a menudo, los parámetros más importantes. Por lo general, se considera que estos índices deben ser superiores a 0,80 para considerar que un procedimiento de cribado es recomendable, pero la interpretación de esos valores psicométricos supone un desafío profesional. Para la interpretación de estos parámetros se necesita un enfoque más cualitativo y estos valores deben interpretarse solo como datos con carácter orientativo y no han de considerarse los únicos elementos en los que basar una recomendación. Es deseable que el sistema de cribado tenga una alta sensibilidad (Coonrod y Stone, 2005; Pinto-Martin y Levy, 2004), aunque no tanta como para sobrecargar en exceso los, ya por lo general saturados, servicios especializados de diagnóstico y de atención temprana (Canal-Bedia et al., 2013). Sensibilidad y especificidad son parámetros interrelacionados, y debe existir equilibrio entre ellos, de tal modo que, si se hacen cambios en los criterios o el punto de corte de la prueba para mejorar la sensibilidad, en realidad se verán afectados ambos índices. 
Falsos positivos y los falsos negativos

Un programa de cribado con una alta sensibilidad a menudo tendrá una alta tasa de falsos positivos, en detrimento de su especificidad. La selección de métodos con una alta especificidad por lo general sacrificará la sensibilidad, implicando un alto número de falsos negativos con la resultante pérdida que eso supondría (retraso en el comienzo de la intervención temprana, peor pronóstico, etc.). Por otro lado, una baja especificidad, con mayor sensibilidad, también tendría consecuencias negativas pues ocasionará una tasa muy alta de falsos positivos. Estos falsos positivos serían evaluados por procedimientos costosos, además del estrés innecesario que se produce en la familia por alarmar falsamente a los padres. Este hecho lleva a la necesidad de dotar al instrumento de cribado de algún recurso de control adicional que permita una mayor precisión antes de recomendar la derivación hacia los servicios especializados de diagnóstico y atención temprana. Haciendo una interpretación más comprensiva de estos valores, igualmente, es crucial considerar la proporción de los casos falsos positivos que, no teniendo TEA, tienen un retraso u otro trastorno del desarrollo y que no han sido previamente detectados (Canal-Bedia et al., 2011). Este tema plantea la cuestión de si es mejor que el programa de cribado vaya dirigido exclusivamente a detectar niños con TEA o a identificar también retrasos evolutivos u otros trastornos del neurodesarrollo en general (Charman y Gotham, 2013). Así, en vez de rechazar inmediatamente un programa de cribado debido a una alta tasa de falsos positivos, una mirada más en profundidad puede indicar que el programa de cribado es provechoso para la detección de niños sin TEA, pero que también se beneficiarían de una evaluación diagnóstica e intervención especializada a una edad temprana.

\section{Prevalencia y valor predictivo positivo}

Otra de las medidas que se consideran más relevantes en un programa de cribado es el valor predictivo positivo (VPP) (Camp, 2006), pero es un índice que depende de la prevalencia de la enfermedad en la población cribada. Esta consideración resalta la importancia de conocer la tasa de prevalencia de TEA en la población de estudio, en vez de establecer su valor en base a los resultados de estudios de prevalencia, pues muchos de esos estudios están realizados en población de más edad (Akobeng, 2007). Un método para calcular la validez de un instrumento de cribado teniendo en cuenta la prevalencia es el Teorema de Bayes. Según este teorema, la posibilidad de que una enfermedad esté realmente presente depende tanto del predominio de la enfermedad como de las propiedades de la prueba, esencialmente la razón de probabilidad (Likelihood ratio) (Akobeng, 2007; Groen, Swinkels, van der Gaag y Buitelaar, 2007). Esto subraya la importancia de indicar claramente la prevalencia utilizada en cada estudio de validez de una herramienta y el uso de las razones de probabilidad. Dado que la prevalencia del autismo en la población general es muy baja (Baird et al., 2006), Groen y cols. sugirieron que una forma de solucionar este problema era administrar la herramienta de cribado sólo a niños previamente identificados como de riesgo dentro de un programa de vigilancia rutinaria del desarrollo (Groen et al., 2007).

(C) Ediciones Universidad de Salamanca

Siglo Cero, vol. 46 (2), n. ${ }^{\circ} 254,2015$, abril-junio, pp. 31-55 


\section{La variabilidad en la población objeto de cribado}

Edad de cribado

La detección de autismo a una edad muy temprana no está exenta de muchas dificultades, ya que puede ser difícil diferenciar TEA de otros trastornos del desarrollo (Charman y Baird, 2002) o incluso a la hora de diferenciar TEA de desarrollo típico (Willemsen-Swinkels, Buitelaar y van Engeland, 2001). Por ejemplo, las conductas repetitivas suelen estar también presentes en los niños pequeños con desarrollo típico (Thelen, 1981). Más aún, muchas de las habilidades de atención conjunta, tales como el contacto visual y actos protodeclarativos, se desarrollan gradualmente desde los 9 hasta los 18 meses en niños de desarrollo típico (Carpenter, Nagell y Tomasello, 1998), y son sólo un signo clínico claro cuando éstos no han aparecido después de la edad de 18 meses. Las dificultades en la distinción de los TEA frente a otros trastornos del desarrollo a una edad muy temprana quedaron patentes en el programa de cribado realizado en Holanda con el ESAT a los 14 meses (Dietz, Swinkels, van Daalen, van Engeland y Buitelaar, 2006) y con el CESDD en Bélgica (Dereu et al., 2010). Ambos estudios son consistentes con el hecho ampliamente extendido en la literatura sobre los signos tempranos de TEA que indica que más de un 30\% de los niños muestran una regresión después de un periodo de desarrollo normal (Chawarska et al., 2007). Por tanto, es probable que los casos más leves de TEA y/o niños con nivel de desarrollo cognitivo alto podrían pasar desapercibidos a una edad muy temprana (Dietz et al., 2006). Por tanto es muy importante considerar la edad de la muestra en el momento de la detección y diagnóstico.

\section{Desarrollo cognitivo y severidad del trastorno}

Los estudios que muestran resultados con diferentes herramientas de cribado coinciden también en la influencia que tiene el CI sobre la sensibilidad de estas herramientas. Esto se refleja en el hecho de que los niños sin retraso intelectual asociado son también más difíciles de detectar, como se demuestra en diversos estudios (Kleinman et al., 2007; Oosterling et al., 2010; Scambler, Rogers y Wehner, 2001).

\section{Las herramientas para la detección}

Variabilidad en función del entorno. Hay estudios que manifiestan haber utilizado una herramienta de cribado de TEA de un modo diferente a como fue concebida inicialmente, adaptando el protocolo original a sus propias necesidades y/o contexto. Por ejemplo, Dereu y cols. (Dereu et al., 2012) no utilizan la entrevista telefónica confirmatoria del M-CHAT que está prevista en el protocolo desarrollado por los autores originales del estudio y, por ejemplo, tanto Nygren y cols. como Canal-Bedia y cols. sí aplican la entrevista telefónica siguiendo el protocolo original de aplicación

(C) Ediciones Universidad de Salamanca

Siglo Cero, vol. 46 (2), n. ${ }^{\circ}$ 254, 2015, abril-junio, pp. 31-55 
del M-CHAT (Canal-Bedia et al., 2011; Nygren et al., 2012). Hoy se piensa que, en el caso en que los niños fallan ocho o más ítems del M-CHAT, la entrevista telefónica complementaria podría ser innecesaria (Chlebowski, Robins, Barton y Fein, 2013) y esos niños podrían ser remitidos directamente una evaluación diagnóstica. Así mismo, el protocolo de entrevista telefónica se puede sustituir por una confirmación inmediata en la consulta del pediatra si éste dispusiera de la ayuda de un ordenador que le ayude a seguir los algoritmos de decisión, tal como ha sido probado en España (García Primo et al., 2013).

Otro ejemplo de adaptación de una herramienta de cribado de autismo lo podemos encontrar en el estudio de Oosterling y cols. de 2009, donde, en vez de utilizar el CHAT como un instrumento independiente, se utilizan ítems del SCQ (Rutter, Bailey y Lord, 2003) y del CSBS-DP (Wetherby, Allen, Cleary, Kublin y Goldstein, 2002) para representar los ítems del CHAT (Oosterling et al., 2009).

\section{Puntos de corte}

En vez de continuar desarrollando continuamente nuevas herramientas de cribado de TEA (existen más de 30 en la actualidad) (ver García-Primo y cols. en prensa), podría ser provechosa una evaluación más exhaustiva de las herramientas ya desarrolladas. Para ello, es útil la exploración de diferentes puntos de corte y muestras de sujetos. Por ejemplo, Oosterling y cols. (2009) exploraron varios puntos de corte del SCQ (Oosterling et al., 2009). Así mismo, en el estudio del M-CHAT en España, el análisis exhaustivo de los resultados planteó que se podría reducir el número de falsos positivos si se incrementara el punto de corte a 5 ítems fallados (Canal-Bedia et al., 2011) frente a 3 que se recomiendan en el estudio original (Robins, Fein, Barton y Green, 2001), sin aumentar el número de falsos negativos.

Ítems

Otra de las diferencias entre las herramientas de cribado es el tipo de ítems que incluyen para identificar la sospecha de posible autismo. Las dificultades sociocomunicativas son consideradas centrales dentro de la sintomatología de los TEA (American Psychiatric Association, 2000) y siempre forman parte de este tipo de herramientas. El ítem "falta de atención conjunta” fue uno de los ítems que resultaron más eficaces en la distinción entre TEA y no TEA en los estudios con el CESDD y con el CHAT (Oosterling et al., 2010; Baron-Cohen, Allen y Gillberg, 1992). Sin embargo, no todas las herramientas incluyen ítems referidos a comportamientos repetitivos y/o hipersensibilidad aunque hay estudios que demuestran la presencia de estos indicadores en edades muy tempranas (Baranek, 1999; Ozonoff et al., 2008). $\mathrm{El}$ hecho de que estos indicadores no hayan sido incluidos en todas las herramientas de cribado podría tener relación con que los padres no suelen mencionar estos ítems espontáneamente. Sin embargo, cuando se les pregunta expresamente a los padres

(C) Ediciones Universidad de Salamanca

Siglo Cero, vol. 46 (2), n. ${ }^{\circ}$ 254, 2015, abril-junio, pp. 31-55 
sobre ello, indican que sí habían notado anormalidades de este tipo desde muy temprana edad (Swinkels et al., 2006). Esto indica la importancia de ampliar el abanico de ítems incluidos. Podría resultar útil por ejemplo ampliar el número de conductas relacionadas con el juego a una edad temprana, mientras que, a partir de los dos años, los indicadores más específicos de TEA serían deficiencias en la interacción social y la comunicación en general. Las diferencias en la formulación de los ítems también pueden afectar a las respuestas. Baird y cols. (2000) (Baird et al., 2000) señalan, por ejemplo, que en su estudio del CHAT la sensibilidad del instrumento podría ser mayor si se preguntaba si el niño "nunca” había producido determinado comportamiento en vez de si "rara vez” lo había hecho, aunque ese incremento de la sensibilidad era a costa del VPP y de la especificidad.

\section{La tasa de participación de los padres}

Aunque los padres no son expertos en la detección de déficits específicos o de TEA, sí suelen acertar en el descubrimiento de un problema del desarrollo (Glascoe, 1997). Dado que los cuestionarios para padres, como el M-CHAT, son fáciles y rápidos de administrar, éstos son a menudo los más utilizados. Sin embargo, muchas veces los padres pueden no saber exactamente qué habilidades deben aparecer en cada cierta edad y a veces no tienen otros niños a su alrededor con los que comparar a su hijo (Dereu et al., 2012) por lo que pueden cometer errores subestimando o sobreestimando los comportamientos que observen en sus hijos. Así, por ejemplo, en el estudio con el ESAT (Dietz et al., 2006), que utiliza a educadores de escuelas infantiles como informantes además de a padres, se comprobó que los primeros analizaban el comportamiento de los niños más negativamente que lo hacían sus padres, de modo que 3 de los 18 niños diagnosticados con TEA hubieran quedado por debajo del punto de corte del ESAT si sólo lo hubieran respondido los padres. Por lo tanto, es importante combinar la información de los padres con las observaciones de un profesional, ya sea educador o pediatra. Por otro lado, la tasa de participación de los padres es a menudo un problema en los estudios de cribado. Por tanto, puede resultar útil examinar las diferencias entre los padres colaboradores y no colaboradores que nos facilite información sobre las razones para no querer participar. En primer lugar, se ha demostrado que los padres de los niños cuyos comportamientos atípicos sean más evidentes muestran una tasa de participación más alta y puntuaciones más altas en el cuestionario (Dietz et al., 2006). Además, los padres menos colaboradores solían tener, en la mayor parte de las ocasiones, niños con menos edad respecto a los no colaboradores (Dereu et al., 2012) y parecían no tener todavía ninguna preocupación. Una posible solución, entonces, podría ser preguntar de nuevo a esos padres cuando hayan pasado unos meses. Dereu y cols. (Dereu et al., 2010), tras realizar su estudio, sugieren que hay que hacer un seguimiento más cercano de estos padres y no darles simplemente un cuestionario y pedirles que te lo devuelvan otro día sin más (Dereu et al., 2010). Otro elemento facilitador podría ser limitar el número de sesiones requeridas para la evaluación diagnóstica en las que es preciso el desplazamiento del niño y la

(C) Ediciones Universidad de Salamanca

Siglo Cero, vol. 46 (2), n. ${ }^{\circ}$ 254, 2015, abril-junio, pp. 31-55 
familia. También, los factores socioeconómicos y culturales podrían estar influyendo (Reznick, Baranek, Reavis, Watson y Crais, 2007), en relación con el miedo al estigma asociado a un posible diagnóstico de autismo (Dyches, Wilder, Sudweeks, Obiakor y Algozzine, 2004).

\section{La participación y formación de los profesionales}

En el Reino Unido, las directrices sobre detección precoz (National Institute for Health and Clinical Excellence-NICE-, 2011) recomiendan el entrenamiento a profesionales sobre los signos tempranos de TEA. Es importante tomar conciencia de que el entrenamiento a profesionales sanitarios y otros profesionales en el reconocimiento temprano de los signos de TEA puede marcar la diferencia en los resultados de los programas de cribado de autismo. El proyecto DIANE en los Países Bajos (Oosterling et al., 2010) es un buen ejemplo de programa de formación a profesionales sanitarios. En general, los resultados de este estudio controlado apoyan el hecho de que la disponibilidad de una herramienta de cribado, combinada con la implicación de los profesionales sanitarios en un programa de formación estructurado sobre signos tempranos de TEA, facilita el proceso de detección-derivación y diagnóstico de este tipo de trastornos. Además, la falta de entrenamiento, unida a la petición de entregar un cuestionario a los padres únicamente, podría conducir a un desacuerdo por su parte con normas impuestas desde fuera, así como desconocimiento sobre los resultados y miedo a la posibilidad de que alguno de los niños que ellos atienden pudiera resultar positivo en la herramienta. Al igual que la sola participación de los padres no es suficiente, la opinión clínica de los pediatras sobre lo que observan acerca de un niño no puede considerarse tampoco la única fuente de información para determinar la sospecha de autismo de un niño, pues el comportamiento de éste en una consulta puede no ser representativo en absoluto de su comportamiento en el contexto habitual. Una manera de solucionar la necesidad de combinar estos dos informantes (padres y profesionales sanitarios) sería implantar los programas de cribado dentro de las escuelas infantiles, donde los educadores pueden observar y comparar con iguales el comportamiento de los niños durante largos periodos de tiempo pero esto sólo sería posible en lugares donde la mayor parte de los niños acudan a escuelas infantiles antes de los tres años, como, por ejemplo, demuestra el estudio realizado en Holanda (Branson, Vigil y Bingham, 2008; Dereu et al., 2010).

\section{Las características del contexto}

Un programa de cribado no puede ser puesto en marcha sin considerar detenidamente todas las características del contexto en el que se va a implantar. La presencia del programa del "Niño sano", en España, por ejemplo, es un marco incomparable frente a países donde no cuentan con dicho programa y donde se limitan a administrar herramientas de cribado a niños ya considerados de riesgo por otras vías (García Primo et al., 2013; García-Primo et al., 2014). Es importante valorar si este tipo de programas está

(C) Ediciones Universidad de Salamanca

Siglo Cero, vol. 46 (2), n. ${ }^{\circ}$ 254, 2015, abril-junio, pp. 31-55 
disponible a toda la población y si cubre familias de todos los grupos socioeconómicos y culturales. Además, en España es fundamental la coordinación entre el sistema de salud y los centros de atención temprana (García Primo et al., 2013). No se debe implantar ningún programa de cribado si no está asegurado el servicio de evaluación diagnóstica y atención temprana de forma inmediata (Canal-Bedia et al., 2013). Además, la coordinación con tales servicios es también crucial para identificar los posibles falsos negativos (García Primo et al., 2013). Por otro lado, si se decide utilizar la herramienta CESDD como herramienta de cribado de autismo, pensada para ser utilizada en escuelas infantiles, se debería garantizar la asistencia de la mayor parte de los niños de ese lugar a este tipo de escuelas, como por ejemplo sucede en Bélgica donde más del 99\% de la población infantil acude desde los primeros meses (Dereu et al., 2010).

\section{La vigilancia del desarrollo}

Uno de los últimos trabajos publicados hasta el momento sobre detección precoz de TEA a nivel poblacional es el "Social Attention and Communication Study (SACS)", desarrollado en el estado de Victoria (Australia) por Barbaro y Dissanayake (Barbaro y Dissanayake, 2010). Consiste en un programa de vigilancia del desarrollo para ser aplicado a los 8, 12, 18 y 24 meses a través del servicio público de enfermería del Programa de Salud Materno-Infantil de Atención Primaria, utilizando una herramienta para detectar autismo a nivel poblacional (nivel 1). Los resultados de este estudio con 22.168 niños indican un valor predictivo positivo del $81 \%$ y unos valores de sensibilidad y especificidad estimados de $83.8 \%$ y $99.8 \%$, especialmente en las edades comprendidas entre los 12 y los 24 meses. La conclusión de este estudio fue que la vigilancia del desarrollo de las conductas sociales y comunicativas permite la identificación precisa de la mayor parte de los niños con riesgo de TEA a estas edades. Así mismo, en los últimos resultados publicados sobre las mejoras introducidas en el programa de cribado de TEA que lleva en estudio más de 8 años con la introducción del programa informático (García Primo et al., 2013), se concluye que la combinación de la vigilancia del desarrollo mediante el entrenamiento de profesionales en la identificación de señales de alerta de TEA, junto con el uso de una herramienta sensible y específica en el contexto poblacional (p. ej., el M-CHAT), mejorará los resultados obtenidos y la escalabilidad del programa.

\section{Búsqueda de biomarcadores}

Queda un largo camino por recorrer en la búsqueda de indicadores tempranos de TEA, tanto comportamentales como biológicos o "biomarcadores". Esta última línea tiene menos tradición pero se tiene la expectativa de que los biomarcadores que se identifiquen antes de la aparición de los síntomas del autismo ayudarán en la detección temprana. En general, la evidencia disponible sugiere que antes de la expresión de los síntomas comportamentales del autismo (en torno al final del

(C) Ediciones Universidad de Salamanca

Siglo Cero, vol. 46 (2), n. ${ }^{\circ}$ 254, 2015, abril-junio, pp. 31-55 
primer año de vida), los datos de funcionamiento del cerebro pueden distinguir entre niños de alto y bajo riesgo para el autismo (Elsabbagh y Johnson, 2010). En teoría, un biomarcador puede contribuir directamente a la susceptibilidad, pero también puede representar un endofenotipo o un rasgo hereditario subyacente indispensable para la susceptibilidad de TEA (Gottesman y Gould, 2003). También puede ocurrir que un biomarcador sea resultado secundario del TEA. Es importante tener en cuenta estas diferentes posibilidades, ya que según sea el planteamiento así será la selección de los objetivos para el diagnóstico y para la intervención. En la Tabla 1 se recogen los biomarcadores que se proponen actualmente para la detección temprana del autismo.

\begin{tabular}{|l|l|}
\hline \multicolumn{2}{|c|}{ TABLA 1. Biomarcadores que se proponen actualmente para el autismo } \\
\hline \multicolumn{1}{|c|}{ Biomarcador } & \multicolumn{1}{c|}{ Método de análisis } \\
\hline $\begin{array}{l}\text { Hiperserotonemia (Azmitia, Singh y } \\
\text { Whitaker-Azmitia, 2011; Hanley, Stahl y } \\
\text { Freedman, 1977; Mulder } \text { et al., 2004) }\end{array}$ & Plasma sanguíneo \\
\hline $\begin{array}{l}\text { Disfunción mitocondrial/inmunológica } \\
\text { (Frye y Rossignol, 2011) }\end{array}$ & Estudio de los linfocitos \\
\hline $\begin{array}{l}\text { Alteración del tamaño de regiones cerebrales } \\
\text { (Kim et al., 2010) }\end{array}$ & $\begin{array}{l}\text { Imagen de Resonancia Magnética (MRI), } \\
\text { Imagen de Tensor de Difusión (DTI) }\end{array}$ \\
\hline $\begin{array}{l}\text { Macrocefalia (Courchesne et al., 2001; Courchesne, } \\
\text { Carper y Akshoomoff, 2003) }\end{array}$ & Trayectoria del perímetro cefálico \\
\hline $\begin{array}{l}\text { Disfunción/hipo-conectividad cerebral } \\
\text { (Barnea-Goraly, Lotspeich y Reiss, 2010; } \\
\text { Shukla, Keehn, Lincoln y Müller, 2010) }\end{array}$ & $\begin{array}{l}\text { Resonancia Magnética Funcional, EEG, } \\
\text { Potenciales Evocados }\end{array}$ \\
\hline Movimiento de los ojos (Dalton et al., 2005) & $\begin{array}{l}\text { Medidas de seguimiento visual, movimientos } \\
\text { sacádicos }\end{array}$ \\
\hline
\end{tabular}

El estudio de biomarcadores es muy prometedor, pero no está exento de múltiples limitaciones que deben superarse antes de que sea posible su uso en el campo de la clínica. En primer lugar, la sensibilidad y especificidad de los biomarcadores propuestos como candidatos a predictores del autismo, probablemente, será muy diferente cuando se apliquen a la población general en estudios poblacionales. En segundo lugar, los estudios hasta ahora publicados usan medidas de laboratorio y se ha sugerido que estas medidas son muy sensibles a la edad (Chawarska y Shic, 2009). Por último, el valor de los biomarcadores propuestos como predictores del autismo se basa en el supuesto de que las diferencias observadas en los niños de riesgo genético para el autismo (por ejemplo hermanos) son necesariamente un "reflejo" de la sintomatología que después presentarán aquellos niños que desarrollen un TEA. Pero esta hipótesis ha sido puesta en cuestión por resultados de estudios que sugieren que el proceso del desarrollo neurológico de niños de riesgo es atípico.

(C) Ediciones Universidad de Salamanca

Siglo Cero, vol. 46 (2), n. ${ }^{\circ}$ 254, 2015, abril-junio, pp. 31-55 


\section{La atención temprana en niños con TEA}

La detección precoz no tiene ningún sentido si no es para iniciar un tratamiento temprano que incluya acciones con el niño, los padres y el entorno cercano a la familia. Esta intervención temprana debe ser individualizada y, en la medida de las necesidades de cada caso, debe ser multidimensional y multidisciplinar. Los objetivos básicos han de dirigirse a desarrollar al máximo la independencia funcional y la calidad de vida del individuo y de su familia a través del aprendizaje, la mejora de las habilidades sociales y de la comunicación, la reducción de la discapacidad y la comorbilidad, la promoción de la independencia y la prestación de apoyos a la familia. Además, desde la detección los niños con TEA deben recibir apoyo para que puedan desarrollar también las áreas donde tiene mayor potencial. Aunque parezca paradójico, ya que el autismo tiene un origen biológico, las intervenciones más eficaces son por el momento las de carácter educativo, teniendo la terapia farmacológica un papel secundario hasta ahora.

Como se ha visto en la sección anterior, la detección precoz ha avanzado de manera notable durante los últimos 15 años. Hoy es posible diagnosticar con un cierto grado de fiabilidad hacia los 24 meses de edad y es posible detectar casos de TEA en torno a los 18 meses, avanzando la detección, no sin esfuerzo, hacia edades aún más tempranas, como los 12 meses o antes. Sin embargo, son aún muy pocas las experiencias exitosas reflejadas en estudios sistemáticos que validen empíricamente procedimientos de tratamiento temprano para niños con TEA menores de 3 años, y aún mucho más escasos son los estudios de tratamiento para niños con TEA menores de 18 meses. La mayoría de las experiencias publicadas en la literatura se refieren, por tanto, a tratamientos dirigidos a niños mayores de 3 años y son muy pocas las que incluyen a niños de 24 meses o menos.

En lo que llevamos del siglo XXI se han publicado diversas revisiones sistemáticas y estudios de metaanálisis sobre la eficacia de los programas de atención temprana en TEA. Lo cual ha sido posible porque en los últimos 15 años han proliferado los estudios sobre resultados de tratamientos para niños pequeños con autismo. Se ha pasado de que en 1998 no hubiera ningún ensayo aleatorio controlado sobre tratamiento de atención temprana en TEA (Rogers y Vismara, 2008), a que en 2013 haya catorce estudios, ocho de los cuales han sido publicados en los últimos dos años, lo que da una idea del crecimiento acelerado que se está dando en este aspecto de la investigación en el autismo temprano. Ya hoy es más difícil sostener la idea de que no hay evidencia suficiente sobre la eficacia de la atención temprana en el autismo, aunque los estudios con rigor metodológico sobre eficacia de los tratamientos se centran en un grupo muy reducido de modelos de intervención.

Siguiendo la tradición sobre evaluación de programas de intervención en el campo del autismo, los programas pueden agruparse en tres categorías: a) Programas de intervención temprana intensiva integral o global; b) programas dirigidos a tratar síntomas o dificultades específicas; y c) programas dirigidos a formar a los padres como terapeutas. 


\section{Los programas integrales de atención temprana}

Los programas integrales de atención temprana constituyen un conjunto de técnicas cuyo objetivo es lograr un gran impacto, amplio y global, abordando los comportamientos más propios del autismo. Estos tratamientos se caracterizan por su intensidad, que implica la dedicación de gran cantidad de tiempo y recursos (por ejemplo, 25 o 30 horas a la semana durante un año o dos) y normalmente tienen varios componentes (Odom, Boyd, Hall y Hume, 2010). Estos programas intensivos integrales suelen incorporar un conjunto de técnicas de intervención específicas, organizadas dentro de un marco teórico conceptual (Rogers y Vismara, 2008) (ej. Modelo Early Start Denver, Modelo Lovaas, etc.). Estos programas aportan datos sobre eficacia obtenidos empíricamente y también manuales de tratamiento, por lo general bien escritos, que especifican tanto el contenido de lo que se debe enseñar como los procedimientos de enseñanza a aplicar. Sin embargo, la aplicación de paquetes integrales e intensivos de tratamiento no siempre permite determinar cuál es el "elemento activo" que hace que el niño progrese. También los tratamientos de marca implican el riesgo asociado a la publicidad y la comercialización. En no pocas ocasiones el acceso a este tipo de tratamientos que han sido publicitados como eficaces se convierte en una pesadilla económica para las familias y el hecho de que sean tratamientos demandados facilita que muchos centros o recursos que ofertan servicios para niños pequeños con autismo tengan la tentación de ofertar este tipo de programas sin la preparación suficiente, lo que afecta a las posibilidades reales de mejoría de los niños. Finalmente, el asociar un modelo o marca de intervención a resultados positivos puede llevar a la creencia de que se debe aplicar el programa tal y como se expone en el manual, ignorando las características individuales (puntos fuertes y débiles del niño). Estos programas integrales, aunque están bien fundamentados por evidencia empírica de resultados, no están pensados para cada niño, sino para un niño ideal, que no existe. Así que su aplicación como un todo puede impedir la elaboración y aplicación de un programa de intervención claramente basado en las características del niño al que se debe dirigir.

Hay gran variedad de estudios que han analizado la eficacia de estos programas integrales. Algunos se centran en la eficacia de programas concretos (Dawson et al., 2010; Lydon, Healy y Leader, 2011; Peters-Scheffer, Didden, Mulders y Korzilius, 2010; Roberts et al., 2011; Smith et al., 2010) y otros son revisiones sistemáticas (Magiati, Moss, Charman y Howlin, 2011; Makrygianni y Reed, 2010; Odom et al., 2010; Peters-Scheffer, Didden, Korzilius y Sturmey, 2011; Virués-Ortega, 2010; Warren et al., 2011a). Las conclusiones que se pueden obtener a partir de la revisión de la literatura actual apoyan la idea, ya aportada por estudios realizados hace más de una década, de que los programas que se basan en el análisis conductual aplicado, y especialmente los que son más intensivos, obtienen algunos resultados positivos en las áreas de habilidades cognitivas, comunicación y conducta adaptativa. Es importante señalar, sin embargo, que los resultados varían entre los estudios y de unos niños a otros. No con todos los niños se consiguen los mismos resultados y no se sabe aún qué tipo de niños con TEA se beneficia más de este tipo de programas. Hay algunos

(C) Ediciones Universidad de Salamanca

Siglo Cero, vol. 46 (2), n. ${ }^{\circ}$ 254, 2015, abril-junio, pp. 31-55 
estudios que comparan intervenciones conductuales intensivas con intervenciones eclécticas con niveles de intensidad similares, encontrando que los niños de ambos grupos logran mejoras significativas, no habiendo diferencias significativas en los logros de los niños de cada grupo (Zachor y Curatolo, 2013).

Warren, McPheeters, Sathe y cols. (Warren et al., 2011) han hecho una revisión sistemática de los estudios publicados sobre intervención conductual intensiva (EIBI) durante los años 2000 y 2010 . Encuentran que hay dos modelos integrales que destacan sobre los demás: el modelo de Lovaas y el modelo Denver. Los autores indican que no hay ningún estudio que haya comparado la eficacia de estos dos métodos entre sí y hay pocos datos sobre cuál de los dos podría dar mejor resultado. Los autores de este estudio también sugieren que la formación específica de los padres no necesariamente intensiva puede ser útil para los niños pequeños con TEA en la mejora de la comunicación social y en el uso del lenguaje y, posiblemente, en la reducción de la gravedad de los síntomas. Pero la evidencia de las investigaciones actuales es aún muy limitada.

\section{Los programas de atención temprana dirigidos a objetivos especificos}

Los programas dirigidos a tratar síntomas o dificultades específicas son intervenciones que se centran en el desarrollo de habilidades en áreas determinadas, como ciertas habilidades de comunicación, las habilidades cognitivas o las habilidades sociales o motoras. Por lo general son programas que tienen una duración corta (de 4 a 6 meses), no son intensivos (como mucho 10-12 sesiones semanales) y tratan de desarrollar habilidades concretas como por ejemplo la atención conjunta y el juego simbólico (Gulsrud, Kasari, Freeman y Paparella, 2007), la sincronía interactiva, la comunicación y habilidades de interacción (Wong y Kwan, 2010), la imitación (Ingersoll, 2010) o la adquisición del sistema PECS (Yoder y Lieberman, 2010).

El trabajo de Landa y cols. (Landa, Holman, O’Neill y Stuart, 2011), por ejemplo, es un ensayo aleatorio controlado con dos grupos en el que se demostró que es posible obtener resultados positivos para ambos grupos. El estudio comparó 2 tratamientos con intervenciones similares en intensidad ( 10 horas por semana para cada niño), la relación niño-profesor, horario, formación de los padres (1,5 horas por mes), tiempo de dedicación a enseñar por parte de los padres (38 horas) y estrategias (técnicas) de enseñanza. La diferencia estaba en que en el grupo de tratamiento se enseñaba la sincronía interpersonal, un contenido complementario consistente en enseñar al niño habilidades de imitación social, atención conjunta e intercambio de afecto. El grupo de tratamiento aumentó significativamente sus habilidades de imitación social y alcanzó niveles casi normales de atención conjunta y afecto compartido.

Otro estudio muy relevante sobre un programa específico es el realizado por Ingersoll (Ingersoll, 2010), que consistió en un ensayo aleatorio controlado sobre la intervención en un marco naturalista para enseñar imitación recíproca a niños pequeños con TEA. Se trataba de incrementar, mediante la intervención, la motivación de los niños para relacionarse e imitar a otros. Los niños en los grupos de tratamiento

(C) Ediciones Universidad de Salamanca

Siglo Cero, vol. 46 (2), n. ${ }^{\circ}$ 254, 2015, abril-junio, pp. 31-55 
mostraron un incremento de sus habilidades de imitación tanto espontánea como inducida. Se encontró una relación entre habilidades previas de juego espontáneo y progresos en las habilidades de imitación. Los niños con mejor nivel de juego espontáneo fueron los que mayor progreso alcanzaron en las habilidades de imitación, por lo tanto, el nivel de juego espontáneo puede ser un indicador pronóstico de la respuesta al tratamiento. En otro estudio previo (Whalen, Schreibman y Ingersoll, 2006) se ha constatado que la intervención sobre alguno de los déficits primarios, como por ejemplo la atención conjunta, produce mejoras en imitación, afecto positivo, juego, inicios sociales y lenguaje espontáneo.

También merece la pena destacar el trabajo de Gulsrud, Kasari, Freeman y Paparella (2007), en el que se comparaban las respuestas de los niños a estímulos novedosos durante su participación en dos intervenciones diferentes: una orientada a desarrollar habilidades de atención conjunta y otra a habilidades de juego simbólico. No había grupo de control. Los niños fueron asignados al azar a una de las dos intervenciones (atención conjunta o juego simbólico). El tratamiento duró entre 5 y 8 semanas, durante el cual se presentaban tres estímulos de carácter auditivo y visual, que aparecían mientras el niño se encontraba en la sesión de trabajo individual. Por ejemplo, aparecía una pelota botando y produciendo música, o una araña que se arrastraba por el suelo y hacía ruido. Se comprobó que los niños que habían recibido entrenamiento en atención conjunta respondieron socialmente mejor que los niños con intervención en juego simbólico y compartieron significativamente más miradas coordinadas con el adulto cuando aparecía el estímulo inesperado.

En este ámbito de las intervenciones dirigidas a síntomas o dificultades específicas hay también algunas revisiones sistemáticas, aunque en su mayoría se dirigen a probar la eficacia de técnicas concretas como la comunicación facilitada (van der Meer y Rispoli, 2010), las historias sociales (Kokina y Kern, 2010), el entrenamiento en integración auditiva (Sinha, Silove, Hayen y Williams, 2011), el uso de grabaciones de video (Kagohara, 2010) o la eficacia del sistema PECS (Preston y Carter, 2009). De los resultados sobre estas revisiones se puede concluir que aún es necesaria más evidencia sobre la eficacia de las historias sociales y de las intervenciones sobre comunicación usando el PECS. Sólo hay alguna evidencia sobre el uso de dispositivos generadores de lenguaje y aun estando documentadas las dificultades de procesamiento sensorial de los niños con autismo, sigue existiendo una evidencia muy limitada sobre la eficacia de la terapia de integración sensorial en la intervención temprana. Las revisiones sistemáticas sobre intervenciones sensoriales, como por ejemplo el entrenamiento en integración auditiva, indican que este tipo de intervenciones no tiene ningún beneficio para los niños pequeños con autismo.

\section{Programas de colaboración con madres y padres}

Finalmente, los programas de intervención dirigidos a formar a los padres como terapeutas son también conocidos como intervenciones mediadas por padres y plantean proveer a las familias de las habilidades, el conocimiento y el apoyo que

(C) Ediciones Universidad de Salamanca

Siglo Cero, vol. 46 (2), n. ${ }^{\circ} 254,2015$, abril-junio, pp. 31-55 
necesitan para facilitar el logro de resultados positivos para sus hijos. En este grupo de intervenciones se incluyen algunos programas de marca como el Hanen ("More than Words"), el Earlybird y otras intervenciones de formación de padres dirigidas a objetivos de enseñanza más específicos. Las intervenciones proporcionadas por los padres pueden mejorar la generalización de habilidades, la eficiencia del tratamiento $y$, sobre todo, pueden hacer que aumente el sentimiento de la autoeficacia de los padres. Hay diversos estudios recientes sobre intervenciones mediadas por padres (Carter et al., 2011; Green et al., 2010; Kasari, Gulsrud, Wong, Kwon y Locke, 2010; Oosterling et al., 2010). Los estudios varían en cuanto a la intensidad del tratamiento, sus objetivos (por ejemplo, uno o más de un ámbito concreto del desarrollo) y el tipo de estrategia de tratamiento/enseñanza utilizada. Algunos estudios constatan una reducción del estrés de los padres asociada a mejoras en el comportamiento problemático o un incremento de su autoeficacia vinculado a progresos en habilidades comunicativas por parte del niño (Keen, Couzens, Muspratt y Rodger, 2010; Whittingham, Sofronoff, Sheffield y Sanders, 2009). Un estudio reciente (Kasari et al., 2010) evaluó una intervención de 8 semanas mediada por los padres dirigida a mejorar las habilidades de atención conjunta. En el estudio se constató que la mayor calidad en la participación del padre/cuidador, según la calificación que hacía el terapeuta, predijo la mayor participación del niño en intercambios sociales y menos fijación sobre objetos. También en otro estudio (Aldred, Green, Emsley y McConachie, 2012) se encontró que los actos comunicativos verbales no directivos, como comentarios, declaraciones o reconocimientos del foco de atención del niño, mejoraban los efectos del tratamiento, incrementando las habilidades sociales, de comunicación y lenguaje de los niños. Del mismo modo, un estudio pequeño de línea de base múltiple, sobre la enseñanza de conductas pivote, constató que las mejoras en la capacidad de los padres para poner en práctica técnicas de entrenamiento en respuestas pivote mejora las habilidades de comunicación de los niños.

Hay también estudios que no encuentran que las intervenciones mediadas por los padres produzcan resultados significativamente mejores (ver, por ejemplo, Carter y cols., Green y cols. y Oosterling y cols.). El estudio de Green y cols. (Green et al., 2010) trató de comprobar si la intervención mediada por padres dirigida a mejorar la comunicación social de los niños producía una reducción de los síntomas de TEA mayor que en un grupo de niños que recibió un tratamiento "tradicional” sin intervención mediada por padres, no encontrándose dicho efecto, aunque las interacciones entre padres e hijos se incrementaron y mejoraron en el grupo de tratamiento. Carter y cols. (Carter et al., 2011) llevaron a cabo un estudio aleatorio controlado del programa Hanen con niños con síntomas de autismo y tampoco encontraron ningún efecto superior sobre los resultados en los niños después de 5 meses de tratamiento. Igualmente, Oosterling y cols. (Oosterling et al., 2010) llevaron a cabo un estudio aleatorio controlado de otra intervención mediada por los padres con niños con TEA en edad preescolar, no encontrando que la formación de los padres tuviera efectos superiores al tratamiento en comparación con un grupo de tratamiento habitual.

Las revisiones sistemáticas llevadas a cabo sobre tratamientos mediados por padres (McConachie y Diggle, 2007; Warren et al., 2011b) identifican algunos estudios que

(c) Ediciones Universidad de Salamanca

Siglo Cero, vol. 46 (2), n. 254, 2015, abril-junio, pp. 31-55 
indican ciertos resultados positivos de las intervenciones mediadas por padres. Los resultados incluyen una reducción significativa de los problemas de comportamiento, mejoras en los estilos de crianza y mayor sentimiento de autoeficacia por parte de los padres después del entrenamiento. Pero también hay estudios que no encuentran efectos positivos en comparación con sus grupos de control. Las intervenciones que dotan a los padres de habilidades de comunicación social y control del comportamiento, aunque sean menos intensivas, pueden ser útiles para mejorar la comunicación social, el uso del lenguaje y para reducir la gravedad de los síntomas, lo que redundará en una mejora del funcionamiento familiar. Sin embargo, aunque los programas de entrenamiento a padres logran modificar las conductas de interacción de los padres, es todavía pronto para disponer de datos suficientes que avalen que esos cambios logran mejoras específicas en los niños (Warren et al., 2011).

\section{Conclusiones}

El propósito de este artículo ha sido proporcionar una revisión actualizada de los procedimientos de detección precoz y de atención temprana en el autismo. Se ha tratado de reflejar los aspectos más relevantes que se deducen de las experiencias y estudios llevados a cabo y que deberían ser tomados en consideración para realizar análisis críticos y constructivos de las experiencias de detección presentes y futuras.

La evaluación y eventual selección de un instrumento de cribado debe tener en cuenta las propiedades psicométricas de sensibilidad, especificidad y valor predictivo. Pero el análisis debe considerar otros aspectos, como el modo en que el procedimiento asegura la identificación de los casos falsos positivos y falsos negativos, ya que el hecho de no tener en cuenta estos aspectos puede llevar a resultados erróneos sobre el valor y utilidad de un determinado instrumento o programa. También es importante considerar el dato de prevalencia para poder valorar adecuadamente los índices de valor predictivo positivo y negativo. Otros aspectos importantes en los programas de cribado son la edad de la población sobre la que se aplica el programa, ya que cuanto menor sea la edad más probable resulta, por el momento, confundir casos de TEA con casos de otras alteraciones tempranas del desarrollo, especialmente si esas alteraciones implican una afectación intelectual. En cuanto a la selección de la herramienta de cribado, solo hay un instrumento adaptado a nuestra población y nuestro sistema asistencial, y también hay algunos cuestionarios traducidos que no han sido validados. Es importante tener en cuenta el tipo de ítems que contiene el instrumento, el punto de corte y los criterios o procedimientos que han llevado a determinar dicho punto de corte. La necesidad de lograr la colaboración de los profesionales y las familias, así como la importancia de tener en cuenta el contexto asistencial, son otros factores que se han encontrado relevantes en las experiencias exitosas de detección precoz.

La detección precoz de los TEA está reconocida como actividad asistencial por la administración sanitaria. Se trata de una actividad beneficiosa tanto para las personas afectadas como para las familias y para el propio sistema de prestación de servicios asistenciales tal y como lo acreditan las numerosas publicaciones científicas citadas.

(C) Ediciones Universidad de Salamanca

Siglo Cero, vol. 46 (2), n. ${ }^{\circ}$ 254, 2015, abril-junio, pp. 31-55 
El camino recorrido ha sido muy importante, pero aún queda un gran trecho que recorrer. Ese camino pasa por sensibilizar al sistema asistencial para que considere la detección precoz no como un acto puntual que tiene lugar en un momento dado del desarrollo temprano, sino que, desde la concepción actual de los TEA como alteraciones del neurodesarrollo, plantee la detección con un enfoque activo de vigilancia del desarrollo, en la cual, sin duda, progresivamente se irán incorporando biomarcadores de dicha alteración neuroevolutiva.

En este artículo se ha asumido que la detección precoz es el primer paso de la atención temprana. Los sistemas de detección precoz pueden ayudar mucho a conocer y comprender las manifestaciones y signos tempranos y ayuda, por tanto, a conocer los mecanismos evolutivos que subyacen a la presentación del trastorno, lo cual es de gran relevancia tanto para el desarrollo de nuevas estrategias de intervención y tratamiento como para, a su vez, avanzar en una detección más temprana. La detección es el primer paso a dar para iniciar el diagnóstico temprano, dando lugar a la oportunidad de una intervención precoz, que mejorará el desarrollo de los niños afectados y hará que las familias afronten mejor el problema.

La revisión realizada nos proporciona una visión esperanzadora, ya que cada vez son más frecuentes, sistemáticos y metodológicamente más apropiados los estudios sobre la eficacia de los programas de atención temprana. La clasificación tipológica de los programas de atención temprana permite diferenciar los que son intensivos e integrales, los que se dirigen a objetivos específicos de aprendizaje y los que implican a los padres. A partir del análisis de los programas revisados se puede llegar a las siguientes conclusiones:

En cuanto a la intensidad del tratamiento, los estudios revisados indican claramente que la mayor intensidad, junto con la mayor precocidad, unidas a un tiempo suficiente de aplicación del programa, constituyen un factor determinante para el logro de resultados positivos. En el campo de la atención temprana a niños con TEA no es raro encontrar programas intensivos con un número de sesiones semanales superior a $20-25$, lo que implica grandes costes para la familia si un profesional o un equipo debe hacerse cargo de todo ese tiempo de dedicación. Esto es menos frecuente en nuestro país. Actualmente se tiende a combinar la intervención de especialistas con programas de visitas al domicilio y programas de formación a padres. Por los datos de la revisión realizada, es posible asumir que si los padres incorporan técnicas específicas para mejorar sus interacciones con sus hijos, manteniendo así una tasa más alta de interacciones a lo largo del día, el niño adquirirá o generalizará habilidades sociales o comunicativas, ya que se incrementarán las oportunidades de aprendizaje a lo largo de todo el día.

El hecho de que haya programas integrales con un enfoque global y programas dirigidos a enseñar conductas específicas plantea la cuestión de cuáles son los “principios activos" de la intervención. Las experiencias de intervención desde hace casi dos décadas y las investigaciones producidas a lo largo de los últimos 15 años, aunque no sean concluyentes en cuanto a cuál es el mejor tratamiento, han logrado algunos resultados positivos partiendo de enfoques diferentes, como el del análisis conductual aplicado o el enfoque evolutivo y han abordado muchos y diferentes

(C) Ediciones Universidad de Salamanca

Siglo Cero, vol. 46 (2), n. ${ }^{\circ}$ 254, 2015, abril-junio, pp. 31-55 
objetivos de intervención, algunos de los cuales son considerados síntomas o déficits primarios de autismo, como las dificultades en atención conjunta, en imitación, en juego simbólico o en el desarrollo del lenguaje. Se ha constatado que la intervención sobre déficits primarios produce mejoras en los niños con TEA, lo que sugiere que las áreas de déficit no son independientes entre sí. También se han citado estudios que se dirigen específicamente a enseñar habilidades concretas, como la atención conjunta, el juego simbólico y las habilidades de interacción. La idea que subyace a todos estos programas es que el trabajo dirigido a determinadas características básicas producirá mejoras posteriores en otras áreas importantes del desarrollo. Queda un largo camino para determinar si es más eficaz un enfoque de tratamiento dirigido a un conjunto limitado de comportamientos o habilidades. Se considera necesario dirigir esfuerzos a buscar los ingredientes activos básicos que pueden lograr cambios significativos en el desarrollo del niño con TEA, ya que las intervenciones dirigidas a características específicas supondrían un enfoque más económico en términos de implicación de recursos asistenciales y dedicación de la familia que lo que hoy ocurre con los programas intensivos integrales.

Finalmente, se ha destacado la importancia de la participación de los padres en la atención temprana. Ciertamente, la presencia de un niño con TEA en la familia supone un reto muy grande. Por lo general las familias están dispuestas a superar las dificultades y a colaborar con los servicios. No hay un modelo único para lograr una colaboración eficaz. Algunos proponen dar instrucciones directas a través de manuales, otros abogan por el entrenamiento directo, individual o en grupos pequeños; otros se inclinan por usar el modelado, bien directamente o mediante videos, por ejemplo. Lo que parece claro es que los niños pequeños con TEA logran mejores resultados si los padres están implicados en el proceso de atención temprana. Pero dadas las especiales dificultades evolutivas que presentan los niños con TEA (comunicativas, sociales, de juego, etc.) muchos padres requieren un apoyo continuado para aprender a afrontar las dificultades de su hijo en el medio familiar. Una opción es la formación de los padres como coterapeutas con el objetivo de incrementar la intensidad y continuidad del tratamiento. No se puede decir, a la luz de la evidencia existente, que la intervención de los padres es determinante. Pero está claro que produce mejoras, y está claro que no puede ser la única opción. No hay opciones únicas en el tratamiento precoz, hay un conjunto de estrategias que se pueden usar y que según parece son eficaces cuando se usan coordinadamente. Hay mucho que aprender todavía sobre cuál es el mejor tratamiento precoz. Es probable, no obstante, que unos padres con formación y apoyo, junto a profesionales sensibles y técnicamente capaces, con conocimientos suficientes, puedan lograr mejoras significativas en el desarrollo de los niños con TEA. Hoy podemos asumir sin miedo a cometer un error grave que la intensidad de la intervención técnicamente cualificada, junto a la participación de los padres, desempeñan un papel en la eficacia de los programas de intervención. También está clara la exigencia de que los programas se adapten a las necesidades de cada niño y cada familia, ya que ninguna intervención integral da lugar a los mismos resultados para todos los niños que reciben esos tratamientos.

(C) Ediciones Universidad de Salamanca

Siglo Cero, vol. 46 (2), n. ${ }^{\circ}$ 254, 2015, abril-junio, pp. 31-55 


\section{Referencias bibliográficas}

Aковеng, A. K. (2007). Understanding diagnostic tests 2: likelihood ratios, pre- and post-test probabilities and their use in clinical practice. Acta Paediatrica (Oslo, Norway: 1992), 96 (4), 487-491. DOI:10.1111/j.1651-2227.2006.00179.x.

Aldred, C., Green, J., Emsley, R. y McConachie, H. (2012). Brief Report: Mediation of Treatment Effect in a Communication Intervention for Pre-School Children with Autism. Journal of Autism and Developmental Disorders, 42 (3), 447-454. Dor:10.1007/s10803-0111248-3.

American Psychiatric Association (2000). Diagnostic and statistical manual of mental disorder: $D S M-I V$ (4th ed., text rev.).

Azmitia, E. C., Singh, J. S. y Whitaker-Azmitia, P. M. (2011). Increased serotonin axons (immunoreactive to 5-HT transporter) in postmortem brains from young autism donors. Neuropharmacology, 60 (7-8), 1347-1354. Dor:10.1016/j.neuropharm.2011.02.002.

Baird, G., Charman, T., Baron-Cohen, S., Cox, A., Swettenham, J., Wheelwright, S. y Drew, A. (2000). A screening instrument for autism at 18 months of age: a 6-year followup study. Journal of the American Academy of Child and Adolescent Psychiatry, 39 (6), 694-702. DOI:10.1097/00004583-200006000-00007.

Baird, G., Simonoff, E., Pickles, A., Chandler, S., Loucas, T., Meldrum, D. y Charman, T. (2006). Prevalence of disorders of the autism spectrum in a population cohort of children in South Thames: the Special Needs and Autism Project (SNAP). Lancet, 368 (1474-547X [Electronic]), 210-215. Recuperado a partir de PM: 16844490.

BaraneK, G. T. (1999). Autism during infancy: a retrospective video analysis of sensorymotor and social behaviors at 9-12 months of age. Journal of Autism and Developmental Disorders, 29 (3), 213-224. DOI:10.1023/A:1023080005650.

Barbaro, J. y Dissanayake, C. (2010). Prospective identification of autism spectrum disorders in infancy and toddlerhood using developmental surveillance: The Social Attention and Communication Study. Journal of Developmental E Behavioral Pediatrics, 31 (5), 376-385.

Barnea-Goraly, N., Lotspeich, L. J. y Reiss, A. L. (2010). Similar white matter aberrations in children with autism and their unaffected siblings: a diffusion tensor imaging study using tract-based spatial statistics. Archives of General Psychiatry, 67 (10), 1052-1060. DOI:10.1001/archgenpsychiatry.2010.123.

Baron-Cohen, S., Allen, J. y Gillberg, C. (1992). Can autism be detected at 18 months? The needle, the haystack, and the CHAT. The British Journal of Psychiatry: The Journal of Mental Science, 161, 839-843. DoI:10.1192/bjp.161.6.839.

Branson, D., Vigil, D. C. y Bingham, A. (2008). Community Childcare Providers' Role in the Early Detection of Autism Spectrum Disorders. Early Childhood Education Journal, 35 (6), 523-530. DOI:10.1007/s10643-008-0243-6.

CAmp, B. W. (2006). What the clinician really needs to know: questioning the clinical usefulness of sensitivity and specificity in studies of screening tests. Journal of Developmental and Behavioral Pediatrics, 27 (3), 226-230.

Canal-Bedia, R., García Primo, P., Martín Cilleros, M. V., Guisuraga Fernández, Z., Herráez, M., Guerra Juanes, I., ... De la Paz, M. (2013). Diagnóstico precoz y sistemas de cribado en los trastornos del espectro autista. En F. Alcantud (Ed.), Trastornos del espectro autista. Detección, diagnóstico e intervención temprana (pp. 61-94). Madrid: Pirámide. 
Canal-Bedia, R., García-Primo, P., Martín-Cilleros, M. V., Santos-Borbujo, J., Guisuraga-Fernández, Z., Herráez-García, L., ... Posada de la Paz, M. (2011). Modified checklist for autism in toddlers: cross-cultural adaptation and validation in Spain. Journal of Autism and Developmental Disorders, 41 (1573-3432 [Electronic]), 1342-1351. DOI:10.1007/s10803-010-1163-z.

Carpenter, M., Nagell, K. y Tomasello, M. (1998). Social cognition, joint attention, and communicative competence from 9 to 15 months of age. Monogr. Soc. Res. Child Dev., 63 (0037-976X [Print]), i-143. Recuperado a partir de PM: 9835078.

Carter, A. S., Messinger, D. S., Stone, W. L., Celimli, S., Nahmias, A. S. y Yoder, P. (2011). A randomized controlled trial of Hanen's "More Than Words" in toddlers with early autism symptoms. Journal of Child Psychology and Psychiatry, and Allied Disciplines, 52 (7), 741-752. DOI:10.1111/j.1469-7610.2011.02395.x.

Charman, T. y Baird, G. (2002). Practitioner review: Diagnosis of autism spectrum disorder in 2- and 3-year-old children. Journal of Child Psycholgy and Psychiatry, 43 (0021-9630 [Print]), 289-305. Recuperado a partir de PM: 11944873.

Charman, T. y Gotham, K. (2013). Measurement Issues: Screening and diagnostic instruments for autism spectrum disorders - lessons from research and practice. Child and Adolescent Mental Health, 18 (1), 52-63. Dor:10.1111/j.1475-3588.2012.00664.x.

Chawarska, K., Paul, R., Klin, A., Hannigen, S., Dichtel, L. E. y Volkmar, F. (2007). Parental recognition of developmental problems in toddlers with autism spectrum disorders. Journal of Autism and Developmental Disorders, 37 (1), 62-72. Dor:10.1007/ s10803-006-0330-8.

Chawarska, K. y Shic, F. (2009). Looking but not seeing: atypical visual scanning and recognition of faces in 2 and 4-year-old children with autism spectrum disorder. Journal of Autism and Developmental Disorders, 39 (12), 1663-1672. Dor:10.1007/s10803-009-0803-7.

Chlebowski, C., Robins, D. L., Barton, M. L. y Fein, D. (2013). Large-scale use of the modified checklist for autism in low-risk toddlers. Pediatrics, 131 (4), e1121-1127. DOI:10.1542/peds.2012-1525.

Coonrod, E. E. y Stone, W. L. (2005). Screening for Autism in Young Children. En F. R. Volkmar, R. Paul, A. Klin y D. Cohen (Eds.), Handbook of Autism and Pervasive Developmental Disorders. John Wiley \& Sons, Inc.

Courchesne, E., Carper, R. y Akshoomoff, N. (2003a). Evidence of brain overgrowth in the first year of life in autism. The Journal of the American Medical Association, 290 (3), 337-344. DOI:10.1001/jama.290.3.337.

Courchesne, E., Karns, C. M., Davis, H. R., Ziccardi, R., Carper, R. A., Tigue, Z. D., ... Courchesne, R. Y. (2001). Unusual brain growth patterns in early life in patients with autistic disorder: an MRI study. Neurology, 57 (2), 245-254.

Dalton, K. M., Nacewicz, B. M., Johnstone, T., Schaefer, H. S., Gernsbacher, M. A., Goldsmith, H. H., ... Davidson, R. J. (2005). Gaze fixation and the neural circuitry of face processing in autism. Nature Neuroscience, 8 (4), 519-526. DoI:10.1038/nn1421.

Dawson, G., Rogers, S., Munson, J., Smith, M., Winter, J., Greenson, J., ... Varley, J. (2010). Randomized, Controlled Trial of an Intervention for Toddlers With Autism: The Early Start Denver Model. Pediatrics, 125 (1), e17-e23. Dor:10.1542/peds.2009-0958.

Dereu, M., Raymaekers, R., Warreyn, P., Schietecatte, I., Meirsschaut, M. y Roeyers, H. (2012). Can child care workers contribute to the early detection of autism spectrum disorders? A comparison between screening instruments with child care workers versus parents as informants. Journal of Autism and Developmental Disorders, 42 (1573-3432 [Electronic]), 781-796. DOI:10.1007/s10803-011-1307-9. 
Dereu, M., Warreyn, P., Raymaekers, R., Meirsschaut, M., Pattyn, G., Schietecatte, I. y Roeyers, H. (2010). Screening for autism spectrum disorders in Flemish day-care centres with the checklist for early signs of developmental disorders. Journal of Autism and Developmental Disorders, 40 (10), 1247-1258. DoI:10.1007/s10803-010-0984-0.

Dietz, C., Swinkels, S., Van Daalen, E., Van Engeland, H. y Buitelaar, J. K. (2006). Screening for autistic spectrum disorder in children aged 14-15 months. II: population screening with the Early Screening of Autistic Traits Questionnaire (ESAT). Design and general findings. Journal of Autism and Developmental Disorders, 36 (6), 713-722. DOI:10.1007/s10803-006-0114-1.

Dyches, T. T., Wilder, L. K., Sudweeks, R. R., Obiakor, F. E. y Algozzine, B. (2004). Multicultural issues in autism. Journal of Autism and Developmental Disorders, 34 (2), 211222. DOI:10.1023/B:JADD.0000022611.80478.73.

Elsabbagh, M., Divan, G., Koh, Y. J., Kim, Y. S., Kauchali, S., Marcín, C., ... Fombonne, E. (2012). Global prevalence of autism and other pervasive developmental disorders. Autism Research: Official Journal of the International Society for Autism Research, 5 (3), 160-179. DOI:10.1002/aur.239.

Elsabbagh, M. y Johnson, M. H. (2010). Getting answers from babies about autism. Trends in Cognitive Sciences, 14 (2), 81-87. DoI:10.1016/j.tics.2009.12.005.

Frye, R. E. y Rossignol, D. A. (2011). Mitochondrial dysfunction can connect the diverse medical symptoms associated with autism spectrum disorders. Pediatric Research, $69(5 \mathrm{Pt}$ 2), 41R-7R. DOI:10.1203/PDR.0b013e318212f16b.

García-Primo, P., Hellendoorn, A., Charman, T., Roeyers, H., Dereu, M., Roge, B., ... Canal-Bedia, R. (2014). Screening for autism spectrum disorders: state of the art in Europe. European Child \& Adolescent Psychiatry. DOI:10.1007/s00787-014-0555-6.

García-Primo, P., Santos Borbujo, J., Martín Cilleros, M. V., Martínez Velarte, M., Lleras Muñoz, S., Posada de la Paz, M. y Canal-Bedia, R. (2013). Pervasive developmental disorders screening program in the health areas of Salamanca and Zamora in Spain. Anales de Pediatría (Barcelona, Spain: 2003). Dor:10.1016/j.anpedi.2013.06.030.

Glascoe, F. P. (1997). Parents' Concerns About Children's Development: Prescreening Technique or Screening Test? Pediatrics, 99 (4), 522-528. Dor:10.1542/peds.99.4.522.

Gottesman, I. I. y Gould, T. D. (2003). The Endophenotype Concept in Psychiatry: Etymology and Strategic Intentions. American Journal of Psychiatry, 160 (4), 636-645. DoI: 10.1176/appi.ajp.160.4.636.

Green, J., Charman, T., McConachie, H., Aldred, C., Slonims, V., Howlin, P., ... Pickles, A. (2010). Parent-mediated communication-focused treatment in children with autism (PACT): a randomised controlled trial. Lancet, 375 (9732), 2152-2160. DoI:10.1016/ S0140-6736(10)60587-9.

Groen, W. B., Swinkels, S. H., Van Der Gaag, R. J. y Buitelaar, J. K. (2007). Finding effective screening instruments for autism using bayes theorem. Archives of Pediatrics $\mathcal{E}$ Adolescent Medicine, 161 (4), 415-416. Dor:10.1001/archpedi.161.4.415.

Gulsrud, A. C., Kasari, C., Freeman, S. y Paparella, T. (2007). Children with autism's response to novel stimuli while participating in interventions targeting joint attention or symbolic play skills. Autism: The International Journal of Research and Practice, 11 (6), 535-546. DOI:10.1177/1362361307083255.

Hanley, H. G., Stahl, S. M. y Freedman, D. X. (1977). Hyperserotonemia and amine metabolites in autistic and retarded children. Archives of General Psychiatry, 34 (5), 521531. DOI:10.1001/archpsyc.1977.01770170031002. 
Haznedar, M. M., Buchsbaum, M. S., Hazlett, E. A., Licalzi, E. M., Cartwright, C. y Hollander, E. (2006). Volumetric analysis and three-dimensional glucose metabolic mapping of the striatum and thalamus in patients with autism spectrum disorders. The American Journal of Psychiatry, 163 (7), 1252-1263. DoI:10.1176/appi.ajp.163.7.1252.

Ingersoll, B. (2010). Brief Report: Pilot Randomized Controlled Trial of Reciprocal Imitation Training for Teaching Elicited and Spontaneous Imitation to Children with Autism. Journal of Autism and Developmental Disorders, 40 (9), 1154-1160. Dor:10.1007/s10803-010-0966-2.

KAGOHARA, D. M. (2010). Is video-based instruction effective in the rehabilitation of children with autism spectrum disorders? Developmental Neurorehabilitation, 13 (2), 129-140. DOI:10.3109/17518420903329281.

Kasari, C., Gulsrud, A. C., Wong, C., Kwon, S. y Locke, J. (2010). Randomized controlled caregiver mediated joint engagement intervention for toddlers with autism. Journal of Autism and Developmental Disorders, 40 (9), 1045-1056. DOI:10.1007/s10803-010-0955-5.

Keen, D., Couzens, D., Muspratt, S. y Rodger, S. (2010). The effects of a parent-focused intervention for children with a recent diagnosis of autism spectrum disorder on parenting stress and competence. Research in Autism Spectrum Disorders, 4 (2), 229-241. DOI:10.1016/j.rasd.2009.09.009.

Kim, J. E., Lyoo, I. K., Estes, A. M., Renshaw, P. F., Shaw, D. W., Friedman, S. D., ... DAgER, S. R. (2010). Laterobasal amygdalar enlargement in 6- to 7-year-old children with autism spectrum disorder. Archives of General Psychiatry, 67 (11), 1187-1197. DOI:10.1001/ archgenpsychiatry.2010.148.

Kleinman, J. M., Robins, D. L., Ventola, P. E., Pandey, J., Boorstein, H. C., Esser, E. L., ... FEIN, D. (2007). The modified checklist for autism in toddlers: a follow-up study investigating the early detection of autism spectrum disorders. Journal of Autism and Developmental Disorders, 38 (0162-3257 [Print]), 827-839. Recuperado a partir de PM: 17882539.

Kogan, M. D., Blumberg, S. J., Schieve, L. A., Boyle, C. A., Perrin, J. M., Ghandour, R. M., ... VAN Dyck, P. C. (2009). Prevalence of parent-reported diagnosis of autism spectrum disorder among children in the US, 2007. Pediatrics, 124 (1098-4275 [Electronic]), 13951403. DOI:10.1542/peds.2009-1522.

Kogan, M. D., Blumberg, S. J., Schieve, L. A., Boyle, C. A., Perrin, J. M., Ghandour, R. M., ... Van Dyck, P. C. (2009). Prevalence of Parent-Reported Diagnosis of Autism Spectrum Disorder Among Children in the US, 2007. Pediatrics, 124 (5), 1395-1403. DOI:10.1542/peds.2009-1522.

KokInA, A. y KERN, L. (2010). Social Story interventions for students with autism spectrum disorders: a meta-analysis. Journal of Autism and Developmental Disorders, 40 (7), 812826. DOI:10.1007/s10803-009-0931-0.

Landa, R. J., Holman, K. C., O’Neill, A. H. y Stuart, E. A. (2011). Intervention targeting development of socially synchronous engagement in toddlers with autism spectrum disorder: a randomized controlled trial. Journal of Child Psychology and Psychiatry, and Allied Disciplines, 52 (1), 13-21. Dor:10.1111/j.1469-7610.2010.02288.x.

Lydon, H., Healy, O. y Leader, G. (2011). A comparison of Video Modeling and Pivotal Response Training to teach pretend play skills to children with Autism Spectrum Disorder. Research in Autism Spectrum Disorders, 5 (2), 872-884. DoI:10.1016/j.rasd.2010.10.002.

Magiati, I., Moss, J., Charman, T. y Howlin, P. (2011). Patterns of change in children with Autism Spectrum Disorders who received community based comprehensive interventions in their pre-school years: A seven year follow-up study. Research in Autism Spectrum Disorders, 5 (3), 1016-1027. DoI:10.1016/j.rasd.2010.11.007.

(C) Ediciones Universidad de Salamanca

Siglo Cero, vol. 46 (2), n. ${ }^{\circ}$ 254, 2015, abril-junio, pp. 31-55 
Makrygianni, M. K. y Reed, P. (2010). A meta-analytic review of the effectiveness of behavioural early intervention programs for children with Autistic Spectrum Disorders. Research in Autism Spectrum Disorders, 4 (4), 577-593. DoI:10.1016/j.rasd.2010.01.014.

McConachie, H. y Diggle, T. (2007). Parent implemented early intervention for young children with autism spectrum disorder: a systematic review. Journal of Evaluation in Clinical Practice, 13 (1), 120-129. DoI:10.1111/j.1365-2753.2006.00674.x.

Mulder, E. J., Anderson, G. M., Kema, I. P., De Bildt, A., Van Lang, N. Dj. ... MinderaA, R. B. (2004). Platelet Serotonin Levels in Pervasive Developmental Disorders and Mental Retardation: Diagnostic Group Differences, Within-Group Distribution, and Behavioral Correlates. Journal of the American Academy of Child and Adolescent Psychiatry, 43 (4), 491-499. DOI:10.1097/00004583-200404000-00016.

National Institute for Health and Clinical Excellence (NICE) (2011). Autism: recognition, referral and diagnosis of children and young people on the autism spectrum. Recuperado a partir de http://publications.nice.org.uk/autism-diagnosis-in-children-andyoung-people-cg128.

Nygren, G., Sandberg, E., Gillstedt, F., Ekeroth, G., Arvidsson, T. y Gillberg, C. (2012). A new screening programme for autism in a general population of Swedish toddlers. Res. Developmental Disability, 33 (1873-3379 (Electronic)), 1200-1210. Recuperado a partir de PM: 22502846.

Odom, S. L., Boyd, B. A., Hall, L. J. y Hume, K. (2010). Evaluation of Comprehensive Treatment Models for Individuals with Autism Spectrum Disorders. Journal of Autism and Developmental Disorders, 40 (4), 425-436. DoI:10.1007/s10803-009-0825-1.

Oosterling, I. J., Swinkels, S. H., Van Der Gaag, R. J., Visser, J. C., Dietz, C. y Buitelaar, J. K. (2009a). Comparative analysis of three screening instruments for autism spectrum disorder in toddlers at high risk. Journal of Autism and Developmental Disorders, 39 (6), 897-909. DOI:10.1007/s10803-009-0692-9.

Oosterling, I. J., Swinkels, S. H., Van Der GaAg, R. J., Visser, J. C., Dietz, C. y BuitelaAr, J. K. (2009b). Comparative analysis of three screening instruments for autism spectrum disorder in toddlers at high risk. Journal of Autism and Developmental Disorders, 39 (6), 897-909. DOI:10.1007/s10803-009-0692-9.

Oosterling, I., Visser, J., Swinkels, S., Rommelse, N., Donders, R., Woudenberg, T., ... Buitelaar, J. K. (2010). Randomized Controlled Trial of the Focus Parent Training for Toddlers with Autism: 1-Year Outcome. Journal of Autism and Developmental Disorders, 40 (12), 1447-1458. DoI:10.1007/s10803-010-1004-0.

Oosterling, I. J., Wensing, M., Swinkels, S. H., Van Der GaAg, R. J., Visser, J. C., Woudenberg, T., ... BuitelaAr, J. K. (2010). Advancing early detection of autism spectrum disorder by applying an integrated two-stage screening approach. Journal of Child Psychology and Psychiatry, and Allied Disciplines, 51 (3), 250-258. Dor:10.1111/ j.1469-7610.2009.02150.x.

Ozonoff, S., Macari, S., Young, G. S., Goldring, S., Thompson, M. y Rogers, S. J. (2008). Atypical object exploration at 12 months of age is associated with autism in a prospective sample. Autism: The International Journal of Research and Practice, 12 (5), 457-472. DOI:10.1177/1362361308096402.

Peters-Scheffer, N., Didden, R., Korzilius, H. y Sturmey, P. (2011). A meta-analytic study on the effectiveness of comprehensive ABA-based early intervention programs for children with Autism Spectrum Disorders. Research in Autism Spectrum Disorders, 5 (1), 60-69. DoI:10.1016/j.rasd.2010.03.011. 
Peters-Scheffer, N., Didden, R., Mulders, M. y Korzilius, H. (2010). Low intensity behavioral treatment supplementing preschool services for young children with autism spectrum disorders and severe to mild intellectual disability. Research in Developmental Disabilities, 31 (6), 1678-1684. DoI:10.1016/j.ridd.2010.04.008.

Pinto-Martin, J. y Levy, S. E. (2004). Early Diagnosis of Autism Spectrum Disorders. Current Treatment Options in Neurology, 6 (5), 391-400.

Preston, D. y Carter, M. (2009). A review of the efficacy of the picture exchange communication system intervention. Journal of Autism and Developmental Disorders, 39 (10), 1471-1486. DOI:10.1007/s10803-009-0763-y.

Reznick, J. S., Baranek, G. T., Reavis, S., Watson, L. R. y Crais, E. R. (2007). A parentreport instrument for identifying one-year-olds at risk for an eventual diagnosis of autism: the first year inventory. Journal of Autism and Developmental Disorders, 37 (9), 1691-1710. DOI:10.1007/s10803-006-0303-y.

Roberts, J., Williams, K., Carter, M., Evans, D., Parmenter, T., Silove, N., ... Warren, A. (2011). A randomised controlled trial of two early intervention programs for young children with autism: Centre-based with parent program and home-based. Research in Autism Spectrum Disorders, 5 (4), 1553-1566. DoI:10.1016/j.rasd.2011.03.001.

Robins, D. L., Fein, D., Barton, M. L. y Green, J. A. (2001). The Modified Checklist for Autism in Toddlers: an initial study investigating the early detection of autism and pervasive developmental disorders. Journal of Autism and Developmental Disorders, 31 (2), 131-144.

Rogers, S. J. y Vismara, L. A. (2008). Evidence-based comprehensive treatments for early autism. Journal of Clinical Child and Adolescent Psychology: The Official Journal for the Society of Clinical Child and Adolescent Psychology, American Psychological Association, Division, 53, 37 (1), 8-38. DoI:10.1080/15374410701817808.

RutTer, BAIley y Lord. (2003). The Social Communication Questionnaire (SCQ). Recuperado a partir de http://bestpracticeautism.blogspot.com.es/2011/06/best-practice-review-social.html.

Scambler, D., Rogers, S. J. y Wehner, E. A. (2001). Can the checklist for autism in toddlers differentiate young children with autism from those with developmental delays? Journal of the American Academy of Child and Adolescent Psychiatry, 40 (12), 1457-1463. DOI:10.1097/00004583-200112000-00017.

Shukla, D. K., Keehn, B., Lincoln, A. J. y Müller, R. A. (2010). White matter compromise of callosal and subcortical fiber tracts in children with autism spectrum disorder: a diffusion tensor imaging study. Journal of the American Academy of Child and Adolescent Psychiatry, 49 (12), 1269-1278, 1278.e1-2. DoI:10.1016/j.jaac.2010.08.018.

Sinha, Y., Silove, N., Hayen, A. y Williams, K. (2011). Auditory integration training and other sound therapies for autism spectrum disorders (ASD). En The Cochrane Collaboration e Y. Sinha (Eds.), Cochrane Database of Systematic Reviews. Chichester, UK: John Wiley \& Sons, Ltd. Recuperado a partir de http://onlinelibrary.wiley.com/ DOI/10.1002/14651858.CD003681.pub3/abstract.

Smith, I. M., Koegel, R. L., Koegel, L. K., Openden, D. A., Fossum, K. L. y Bryson, S. E. (2010). Effectiveness of a novel community-based early intervention model for children with autistic spectrum disorder. American Journal on Intellectual and Developmental Disabilities, 115 (6), 504-523. Dor:10.1352/1944-7558-115.6.504.

Swinkels, S. H. N., Dietz, C., Van Daalen, E., Kerkhof, I. H. G. M., Van Engeland, H. y BuitelaAR, J. K. (2006). Screening for autistic spectrum in children aged 14 to 15 months. I: the development of the Early Screening of Autistic Traits Questionnaire (ESAT). Journal of Autism and Developmental Disorders, 36 (6), 723-732. Dor:10.1007/s10803-006-0115-0.

(C) Ediciones Universidad de Salamanca

Siglo Cero, vol. 46 (2), n. ${ }^{\circ}$ 254, 2015, abril-junio, pp. 31-55 
Thelen, E. (1981). Kicking, rocking, and waving: contextual analysis of rhythmical stereotypies in normal human infants. Animal Behaviour, 29 (1), 3-11.

Van Der Meer, L. A. J. y Rispoli, M. (2010). Communication interventions involving speechgenerating devices for children with autism: a review of the literature. Developmental Neurorehabilitation, 13 (4), 294-306. Dor:10.3109/17518421003671494.

Virués-Ortega, J. (2010). Applied behavior analytic intervention for autism in early childhood: meta-analysis, meta-regression and dose-response meta-analysis of multiple outcomes. Clinical Psychology Review, 30 (4), 387-399. DoI:10.1016/j.cpr.2010.01.008.

Warren, Z., McPheeters, M. L., Sathe, N., Foss-Feig, J. H., Glasser, A. y VeenstraVAnderweele, J. (2011a). A Systematic Review of Early Intensive Intervention for Autism Spectrum Disorders. Pediatrics, peds. 2011-0426. DoI: 10.1542/peds.2011-0426.

Warren, Z., McPheeters, M. L., Sathe, N., Foss-Feig, J. H., Glasser, A. y VeenstraVANDERWEele, J. (2011b). A Systematic Review of Early Intensive Intervention for Autism Spectrum Disorders. Pediatrics, peds. 2011-0426. DoI: 10.1542/peds.2011-0426.

Wetherby, A. M., Allen, L., Cleary, J., Kublin, K. y Goldstein, H. (2002). Validity and reliability of the communication and symbolic behavior scales developmental profile with very young children. Journal of Speech, Language, and Hearing Research, 45 (6), 1202 1218.

Whalen, C., Schreibman, L. e Ingersoll, B. (2006). The collateral effects of joint attention training on social initiations, positive affect, imitation, and spontaneous speech for young children with autism. Journal of Autism and Developmental Disorders, 36 (5), 655-664. DOI:10.1007/s10803-006-0108-z.

Whittingham, K., Sofronoff, K., Sheffield, J. y Sanders, M. R. (2009). Stepping Stones Triple P: an RCT of a parenting program with parents of a child diagnosed with an autism spectrum disorder. Journal of Abnormal Child Psychology, 37 (4), 469-480. Dor:10.1007/ s10802-008-9285-X.

Willemsen-Swinkels, S. H., Buitelaar, J. K. y Van Engeland, H. (2001). Is 18 months too early for the CHAT? Journal of the American Academy of Child and Adolescent Psychiatry, 40 (7), 737-738. DOI:10.1097/00004583-200107000-00002.

Wong, V. C. N. y Kwan, Q. K. (2010). Randomized controlled trial for early intervention for autism: a pilot study of the Autism 1-2-3 Project. Journal of Autism and Developmental Disorders, 40 (6), 677-688. Dor:10.1007/s10803-009-0916-z.

Yoder, P. J. y Lieberman, R. G. (2010). Brief Report: Randomized test of the efficacy of picture exchange communication system on highly generalized picture exchanges in children with ASD. Journal of Autism and Developmental Disorders, 40 (5), 629-632. DOI:10.1007/s10803-009-0897-y.

Zachor, D. A. y Curatolo, P. (2013). Recommendations for early diagnosis and intervention in autism spectrum disorders: An Italian-Israeli consensus conference. European Journal of Paediatric Neurology: Official Journal of the European Paediatric Neurology Society.

Declaración de intereses: Los autores manifiestan la inexistencia de conflictos de interés en relación con este artículo.

Agradecimientos: Este artículo se ha realizado en el marco de la investigación financiada por la Consejería de Sanidad de la Junta de Catilla y León, referencia GES/SA04/13. 